\title{
Exact anisotropic brane cosmologies
}

\author{
Chiang-Mei Chen* \\ Department of Physics, National Taiwan University, Taipei 106, Taiwan \\ T. Harko ${ }^{\dagger}$ \\ Department of Physics, The University of Hong Kong, Pokfulam, Hong Kong
}

M. K. Mak

Department of Physics, The Hong Kong University of Science and Technology, Clear Water Bay, Hong Kong

(Received 5 April 2001; published 26 July 2001)

\begin{abstract}
We present exact solutions of the gravitational field equations in the generalized Randall-Sundrum model for an anisotropic brane with Bianchi type I and V geometry, with perfect fluid and scalar fields as matter sources. Under the assumption of a conformally flat bulk (with a vanishing Weyl tensor) for a cosmological fluid obeying a linear barotropic equation of state the general solution of the field equations can be expressed in an exact parametric form for both Bianchi type I and V space-times. In the limiting case of a stiff cosmological fluid with pressure equal to the energy density, for a Bianchi type I universe the solution of the field equations is obtained in an exact analytic form. Several classes of scalar field models of evolution on the brane are also considered, corresponding to different choices of the scalar field potential. For all models the behavior of the observationally important parameters such as shear, anisotropy, and the deceleration parameter is considered in detail.
\end{abstract}

DOI: $10.1103 /$ PhysRevD.64.044013

PACS number(s): 04.20.Jb, 04.65.+e, 98.80.-k

\section{INTRODUCTION}

In two recent papers Randall and Sundrum [1,2] have shown that a scenario with an infinite fifth dimension in the presence of a brane can generate a theory of gravity which mimics purely four-dimensional gravity, both with respect to the classical gravitational potential and with respect to gravitational radiation. The gravitational self-couplings are not significantly modified in this model. This result has been obtained from the study of a single 3-brane embedded in five dimensions, with the 5D metric given by $d s^{2}$ $=e^{-f(y)} \eta_{\mu \nu} d x^{\mu} d x^{\nu}+d y^{2}$, which can produce a large hierarchy between the scale of particle physics and gravity due to the appearance of the warp factor. Even if the fifth dimension is uncompactified, standard 4D gravity is reproduced on the brane. In contrast with the compactified case, this follows because the near-brane geometry traps the massless graviton. Hence this model allows the presence of large or even infinite noncompact extra dimensions. Our brane is identified to a domain wall in a 5-dimensional anti-de Sitter space-time.

The Randall-Sundrum model was inspired by superstring theory. The ten-dimensional $E_{8} \times E_{8}$ Heterotic string theory, which contains the standard model of elementary particles, could be a promising candidate for the description of the real universe. This theory is connected with an elevendimensional theory, $M$ theory, compactified on the orbifold $R^{10} \times S^{1} / Z_{2}$ [3]. In this model we have two separated tendimensional manifolds.

The static Randall-Sundrum solution has been extended to

\footnotetext{
*Email address: cmchen@phys.ntu.edu.tw

†Email address: tcharko@hkusua.hku.hk

‡Email address: mkmak@vtc.edu.hk
}

time-dependent solutions and their cosmological properties have been extensively studied in papers, too many to cite them all [4-11] (for a recent review of dynamics and geometry of brane universes see Ref. [12]). In one of the first cosmological applications of this scenario it was pointed out that a model with a noncompact fifth dimension is potentially viable, while the scenario which might solve the hierarchy problem predicts a contracting universe, leading to a variety of cosmological problems [13]. By adding cosmological constants to the brane and bulk, the problem of the correct behavior of the Hubble parameter on the brane has been solved by Cline, Grojean, and Servant [14]. As a result one also obtains normal expansion during nucleosynthesis, but faster than normal expansion in the very early universe. The creation of a spherically symmetric brane-world in AdS bulk has been considered, from a quantum cosmological point of view, with the use of the Wheeler-deWitt equation, by Anchordoqui, Nuñez, and Olsen [15].

The effective gravitational field equations on the brane world, in which all the matter forces except gravity are confined on the 3-brane in a 5-dimensional space-time with $Z_{2}$-symmetry have been obtained, by using an elegant geometric approach, by Shiromizu, Maeda, and Sasaki $[16,17]$. The correct signature for gravity is provided by the brane with positive tension. If the bulk space-time is exactly anti-de Sitter, generically the matter on the brane is required to be spatially homogeneous. The electric part of the 5-dimensional Weyl tensor $E_{I J}$ gives the leading order corrections to the conventional Einstein equations on the brane. The four-dimensional field equations for the induced metric and scalar field on the world-volume of a 3-brane in the five-dimensional bulk with Einstein gravity plus a selfinteracting scalar field have been derived by Maeda and Wands [18]. The effective four-dimensional Einstein equa- 
tions include terms due to scalar fields and gravitational waves in the bulk.

From a general theoretical point of view brane cosmological models are two-measure theories, since they have two independent Lagrangians, one associated to the brane, the other associated to the bulk. Another two measure theory has been proposed by Guendelman [19], in which the energymomentum tensor is also a nonlinear function of the generalrelativistic energy-momentum tensor. Because of scale invariance, the true vacuum state has zero energy density, when the theory is analyzed in the conformal Einstein frame. Field theory models, constructed in a gravitational theory where a measure of integration $\Phi$ in the action is not necessary $\sqrt{-g}$, but is determined dynamically through additional degrees of freedom, have been presented in Ref. [20]. In this type of theory it is possible to combine the solution of the cosmological constant problem with the possibility of inflation and spontaneously broken gauge unified theories. The models with global scale invariance allow for nontrivial scalar field potentials and masses for particles, so that the scale symmetry must be broken. The conserved quantities have been obtained by Guendelman [21], who showed that the infrared behavior of the conserved currents is singular, so there are no conserved charges associated with scale symmetry (which implies that in some high field region the potentials become flat). For closed strings and branes (including the supersymmetric case) the modified measure formulation is possible and does not require the introduction of a particular scale (the string or brane tension) from the beginning, but rather these appear as integration constants [22].

The linearized perturbation equations in the generalized Randall-Sundrum model have been obtained, by using the covariant nonlinear dynamical equations for the gravitational and matter fields on the brane, by Maartens [23]. The nonlocal energy density determines the tidal acceleration in the off-brane direction and can oppose singularity formation via the generalized Raychaudhuri equation. Isotropy of the cosmic microwave background may no longer guarantee a Friedmann-Robertson-Walker geometry. Vorticity on the brane decays as in general relativity, but nonlocal bulk effects can source the gravitomagnetic field, so that vector perturbations can also be generated in the absence of vorticity.

The behavior of an anisotropic Bianchi type I brane-world in the presence of inflationary scalar fields has been considered by Maartens, Sahni, and Saini [24]. According to their results the magnitude of the anisotropy parameter of the brane does not affect inflation, a large initial anisotropy introducing more damping into the scalar field equation of motion. Inflation at high energies proceeds at a higher rate than the corresponding rate in general relativity. By numerical integration of field equations it is also found that anisotropy always disappears within a fixed interval of time, no matter what its initial value. The shear dynamics in Bianchi type I cosmological model on a brane with a perfect fluid has been studied in Ref. [25]. For $1<\gamma<2$ the shear has a maximum at some moment during a transition period from nonstandard to standard cosmology, when the matter energy density is comparable to the brane tension.

A systematic analysis, using dynamical systems tech- niques, of the qualitative behavior of the Bianchi type I and $\mathrm{V}$ cosmological models in the Randall-Sundrum brane world scenario, with matter on the brane obeying a barotropic equation of state $p=(\gamma-1) \rho$, has been performed by Campos and Sopuerta [26]. In particular, they constructed the state spaces for these models and discussed what new critical points appear, the occurrence of bifurcations and the dynamics of the anisotropy. Expanding Bianchi type I and V braneworlds always isotropize, although there could be intermediate stages in which the anisotropy grows. Near the big-bang the anisotropy dominates for $\gamma \leqslant 1$, in opposition to the general relativistic situation when it dominates for $\gamma<2$. Frolov [27] has shown that the geometrical construction of the Randall and Sundrum brane world can be carried out, without the assumption of spatial isotropy, by means of an homogeneous and anisotropic Kasner type solution of the Einstein-AdS equations in the bulk. The brane equations of motion in this anisotropic space-time are solved by a static brane configuration, with the geometry of the 3-brane given by the $4 \mathrm{D}$ Kasner solution. The set of sufficient conditions, which must be satisfied by the brane matter and bulk metric so that a homogeneous and anisotropic brane asymptotically evolves to a de Sitter space-time in the presence of a positive cosmological constant have been derived in Ref. [28]. In the presence of a nonlocal energy density and/or a strong anisotropic stress (i.e., a magnetic field), a Bianchi type I brane, even initially expanding, is unstable and may collapse.

It is the purpose of this paper to investigate some classes of exact solutions of the gravitational field equations in the brane world model for the anisotropic Bianchi type I and V geometries, for a conformally flat bulk (with vanishing Weyl tensor). For a perfect cosmological fluid obeying a linear barotropic equation of state, the general solution of the field equations can be obtained in an exact parametric form, for arbitrary $\gamma$ or in an exact analytic form for a stiff fluid. The inclusion of the quadratic terms in the energy-momentum tensor of the perfect cosmological fluids leads to major changes in the early dynamics of the anisotropic universe, as compared to the standard general relativistic case. We also consider the dynamics of the scalar fields on the brane for different scalar field potentials of physical interest. The behavior of the observationally important physical quantities such as anisotropy, shear, and deceleration parameters is considered in detail for all these models.

The present paper is organized as follows. The field equations on the brane are written down in Sec. II. In Sec. III we present the general solution of the field equations for the Bianchi type I geometry with a perfect cosmological fluid. Evolution of the scalar fields in the brane-world is considered in Sec. IV. Bianchi type V space-times are investigated in Sec. V. In Sec. VI we discuss and conclude our results.

\section{BRANE GEOMETRY, FIELD EQUATIONS, AND CONSEQUENCES}

In the 5D space-time the brane-world is located as $Y\left(X^{I}\right)=0$, where $X^{I}, I=0,1,2,3,4$ are 5-dimensional coordinates. The effective action in five dimensions is [18] 


$$
\begin{aligned}
S= & \int d^{5} X \sqrt{-g_{5}}\left(\frac{1}{2 k_{5}^{2}} R_{5}-\Lambda_{5}\right)+\int_{Y=0} d^{4} x \sqrt{-g} \\
& \times\left(\frac{1}{k_{5}^{2}} K^{ \pm}-\lambda+L^{\text {matter }}\right),
\end{aligned}
$$

with $k_{5}^{2}=8 \pi G_{5}$ the 5-dimensional gravitational coupling constant and where $x^{\mu}, \mu=0,1,2,3$ are the induced 4 -dimensional brane world coordinates. $R_{5}$ is the 5D intrinsic curvature in the bulk and $K^{ \pm}$is the intrinsic curvature on either side of the brane.

On the 5-dimensional space-time (the bulk), with the negative vacuum energy $\Lambda_{5}$ as only source of the gravitational field the Einstein field equations are given by

$$
G_{I J}=k_{5}^{2} T_{I J}, \quad T_{I J}=-\Lambda_{5} g_{I J}+\delta(Y)\left[-\lambda g_{I J}+T_{I J}^{\text {matter }}\right] .
$$

In this space-time a brane is a fixed point of the $Z_{2}$ symmetry. In the following capital Latin indices run in the range $0, \ldots, 4$ while Greek indices take the values $0, \ldots, 3$.

Assuming a metric of the form $d s^{2}=\left(n_{I} n_{J}+g_{I J}\right) d x^{I} d x^{J}$, with $n_{I} d x^{I}=d \chi$ the unit normal to the $\chi=$ const hypersurfaces and $g_{I J}$ the induced metric on $\chi=$ const hypersurfaces, the effective four-dimensional gravitational equations on the brane take the form $[16,17]$

$$
G_{\mu \nu}=-\Lambda g_{\mu \nu}+k_{4}^{2} T_{\mu \nu}+k_{5}^{4} S_{\mu \nu}-E_{\mu \nu},
$$

where

$$
S_{\mu \nu}=\frac{1}{12} T T_{\mu \nu}-\frac{1}{4} T_{\mu}{ }^{\alpha} T_{\nu \alpha}+\frac{1}{24} g_{\mu \nu}\left(3 T^{\alpha \beta} T_{\alpha \beta}-T^{2}\right),
$$

and $\quad \Lambda=k_{5}^{2}\left(\Lambda_{5}+k_{5}^{2} \lambda^{2} / 6\right) / 2, k_{4}^{2}=k_{5}^{4} \lambda / 6, \quad$ and $\quad E_{I J}$ $=C_{I A J B} n^{A} n^{B} . C_{I A J B}$ is the 5-dimensional Weyl tensor in the bulk and $\lambda$ is the vacuum energy on the brane. $T_{\mu \nu}$ is the matter energy-momentum tensor on the brane with components $T_{0}^{0}=-\rho, T_{1}^{1}=T_{2}^{2}=T_{3}^{3}=p$, and $T=T^{\mu}{ }_{\mu}$ is the trace of the energy-momentum tensor. In this paper we restrict our analysis to a conformally flat bulk geometry with $C_{I A J B} \equiv 0$. The Einstein equation in the bulk implies the conservation of the energy momentum tensor of the matter on the brane

$$
T_{\mu}^{\nu} ;\left.\nu\right|_{\chi=0}=0
$$

In the following we investigate the Bianchi type universes that are obtained from space-time geometries that admit a simple transitive three-dimensional group of isometries $G_{3}$ on spacelike hypersurfaces. Hence the corresponding cosmological models are spatially homogeneous. There is only one essential dynamical coordinate, the time $t$, and the gravitational field equations reduce to ordinary differential equations. The metric on the brane is given in the general case by

$$
d s^{2}=-d t^{2}+\gamma_{a b}(t) e_{i}^{a}(x) d x^{i} e_{j}^{b}(x) d x^{j},
$$

where $e_{i}^{a}(x)$ are one-forms inverse to the spatial vector triad $e_{a}^{i}(x)$, which have the same commutators $C_{b c}^{a}, a, b, c$ $=1,2,3$ as the structure constants of the group of isometries and commute with the unit normal vector $e_{0}$ to the surfaces of homogeneity, that is, $e_{a}=e_{a}^{i} \partial / \partial x^{i}$ and $e_{0}=\partial / \partial t$ obey the commutation relations $\left[e_{a}, e_{b}\right]=C_{a b}^{c} e_{c},\left[e_{0}, e_{a}\right]=0$.

In order to simplify the calculations we only consider geometries so that $\gamma_{a b}(t)$ can be taken to be a diagonal matrix. We denote by $a_{i}(t), \quad i=1,2,3$ the components of the metric tensor on the anisotropic brane.

We define the following variables:

$$
V=\prod_{i=1}^{3} a_{i} \quad \text { (volume scale factor), }
$$

$H_{i}=\frac{\dot{a}_{i}}{a_{i}}, i=1,2,3$ (directional Hubble parameters),

$H=\frac{1}{3} \sum_{i=1}^{3} H_{i}$ (mean Hubble parameter),

$\Delta H_{i}=H_{i}-H, \quad i=1,2,3$.

From Eqs. (7), (8), and (9) we obtain $H=\dot{V} / 3 V$.

The physical quantities of observational interest in cosmology are the expansion scalar $\theta$, the mean anisotropy parameter $A$, the shear scalar $\sigma^{2}$ and the deceleration parameter $q$, all are defined according to

$$
\begin{aligned}
\theta & =3 H \\
A & =\frac{1}{3} \sum_{i=1}^{3}\left(\frac{\Delta H_{i}}{H}\right)^{2}, \\
\sigma^{2} & =\frac{1}{2} \sigma_{i k} \sigma^{i k}=\frac{1}{2}\left(\sum_{i=1}^{3} H_{i}^{2}-3 H^{2}\right)=\frac{3}{2} A H^{2}, \\
q & =\frac{d}{d t} H^{-1}-1=-H^{-2}\left(\dot{H}+H^{2}\right) .
\end{aligned}
$$

The sign of the deceleration parameter indicates whether the model inflates or not. The positive sign of $q$ corresponds to "standard" decelerating models whereas the negative sign indicates inflation.

\section{EVOLUTION OF PERFECT COSMOLOGICAL FLUIDS ON BIANCHI TYPE I BRANE UNIVERSES}

The line element of a Bianchi type I space-time, which generalizes the flat Robertson-Walker metric to the anisotropic case, is given by

$$
d s^{2}=-d t^{2}+a_{1}^{2}(t) d x^{2}+a_{2}^{2}(t) d y^{2}+a_{3}^{2}(t) d z^{2} .
$$

We assume that the thermodynamic pressure $p$ of the cosmological fluid obeys a linear barotropic equation of state $p$ $=(\gamma-1) \rho, \gamma=$ const and $1 \leqslant \gamma \leqslant 2$. 
Using the variables (7)-(9) the gravitational field equations and the Bianchi identity on the brane take the form

$$
\begin{aligned}
& 3 \dot{H}+\sum_{i=1}^{3} H_{i}^{2}=\Lambda-\frac{3 \gamma-2}{2} k_{4}^{2} \rho-\frac{3 \gamma-1}{12} k_{5}^{4} \rho^{2}, \\
& \frac{1}{V} \frac{d}{d t}\left(V H_{i}\right)=\Lambda-\frac{\gamma-2}{2} k_{4}^{2} \rho-\frac{\gamma-1}{12} k_{5}^{4} \rho^{2}, \quad i=1,2,3,
\end{aligned}
$$

$$
\dot{\rho}+3 \gamma H \rho=0 .
$$

Thus, the time evolution of the energy density of the matter is given by

$$
\rho=\rho_{0} V^{-\gamma}, \quad \rho_{0}=\text { const }>0 .
$$

By summing Eqs. (17) we find

$$
\frac{1}{V} \frac{d}{d t}(V H)=\Lambda-\frac{\gamma-2}{2} k_{4}^{2} \rho-\frac{\gamma-1}{12} k_{5}^{4} \rho^{2} .
$$

Subtracting Eq. (20) back to Eqs. (17) we obtain

$$
H_{i}=H+\frac{K_{i}}{V}, \quad i=1,2,3,
$$

with $K_{i}, i=1,2,3$ constants of integration satisfying the consistency condition $\sum_{i=1}^{3} K_{i}=0$. By using the evolution equation of the matter energy density Eq. (20), the basic equation describing the dynamics of the anisotropic brane world for a conformally flat bulk can be written as

$$
\ddot{V}=3 \Lambda V-\frac{3(\gamma-2)}{2} k_{4}^{2} \rho_{0} V^{1-\gamma}-\frac{\gamma-1}{4} k_{5}^{4} \rho_{0}^{2} V^{1-2 \gamma},
$$

and has the general solution

$t-t_{0}=\int\left(3 \Lambda V^{2}+3 k_{4}^{2} \rho_{0} V^{2-\gamma}+\frac{1}{4} k_{5}^{4} \rho_{0}^{2} V^{2-2 \gamma}+C\right)^{-1 / 2} d V$,

where $C$ is a constant of integration.

Therefore for a Bianchi type I induced brane geometry the general solution of the gravitational field equations can be expressed in the following exact parametric form, with $V$ $\geqslant 0$ taken as parameter:

$$
\begin{aligned}
\theta= & \left(3 \Lambda+3 k_{4}^{2} \rho_{0} V^{-\gamma}+\frac{1}{4} k_{5}^{4} \rho_{0}^{2} V^{-2 \gamma}+C V^{-2}\right)^{1 / 2}, \\
a_{i}= & a_{0 i} V^{1 / 3} \exp \left[K _ { i } \int \left(3 \Lambda V^{4}+3 k_{4}^{2} \rho_{0} V^{4-\gamma}\right.\right. \\
& \left.\left.+\frac{1}{4} k_{5}^{4} \rho_{0}^{2} V^{4-2 \gamma}+C V^{2}\right)^{-1 / 2} d V\right], \quad i=1,2,3,
\end{aligned}
$$

$$
\begin{gathered}
A=3 K^{2}\left(3 \Lambda V^{2}+3 k_{4}^{2} \rho_{0} V^{2-\gamma}+\frac{1}{4} k_{5}^{4} \rho_{0}^{2} V^{2-2 \gamma}+C\right)^{-1} \\
\sigma^{2}=\frac{K^{2}}{2} V^{-2}, \\
q=2-\frac{36 \Lambda V^{2}+18(2-\gamma) k_{4}^{2} \rho_{0} V^{2-\gamma}+3(1-\gamma) k_{5}^{4} \rho_{0}^{2} V^{2-2 \gamma}}{12 \Lambda V^{2}+12 k_{4}^{2} \rho_{0} V^{2-\gamma}+k_{5}^{4} \rho_{0}^{2} V^{2-2 \gamma}+4 C},
\end{gathered}
$$

where $K^{2}=\sum_{i=1}^{3} K_{i}^{2}$.

With the use of Eqs. (19), (21), and (24), from Eq. (16) it follows that the arbitrary integration constants $K_{i}, i$ $=1,2,3$ and $C$ must satisfy the consistency condition

$$
K^{2}=\frac{2}{3} C .
$$

At densities significantly greater than the nuclear one $\rho_{n}$, e.g., $\rho \gg \rho_{n}$, we have $p \rightarrow \rho$, with the speed of sound $c_{s}$ tending to the speed of light, $c_{s} \rightarrow c$. A typical approach to the nuclear equation of state in the very high-density regime is to construct a relativistic Lagrangian that allows "bare" nucleons to interact attractively via scalar meson exchange and repulsively via the exchange of a more massive vector meson. But at the highest densities the vector meson exchange dominates and one still has $p=\rho$. Therefore the equation of state most appropriate to describe the high density regime of the early universe is the stiff Zeldovich one, with $\gamma=2$. For a stiff cosmological fluid, the dynamics of the matter on the brane is mainly determined by the correction terms from $S_{\mu \nu}$, quadratic in the matter variables and due to the form of the Gauss-Codazzi equations.

With $\gamma=2$ Eq. (23) becomes

$$
t-t_{0}=\int \frac{V d V}{\sqrt{3 \Lambda V^{4}+\alpha V^{2}+\beta}},
$$

where $\alpha=3 k_{4}^{2} \rho_{0}+C$ and $\beta=\frac{1}{4} k_{5}^{4} \rho_{0}^{2}$. The time dependence of the volume scale factor of the Bianchi type I universe in the high-energy limit is therefore given by

$$
V(t)=\frac{1}{2 \sqrt{3 \Lambda}} e^{-\sqrt{3 \Lambda}\left(t-t_{0}\right)} \sqrt{\left(e^{2 \sqrt{3 \Lambda}\left(t-t_{0}\right)}-\alpha\right)^{2}-12 \Lambda \beta} .
$$

For $t=t_{S}=t_{0}+\ln (2 \sqrt{3 \beta \Lambda}+\alpha) / 2 \sqrt{3 \Lambda}$ the volume scale factor is zero, $V\left(t_{S}\right)=0$. By reparametrizing the initial value of the cosmological time according to

$$
\exp \left(-2 \sqrt{3 \Lambda} t_{0}\right)=\alpha+2 \sqrt{3 \Lambda \beta},
$$

the evolution of the high-density brane universe starts from $t=0$ from a singular state $V_{0}=V(t=0)=0$.

The time evolution of the expansion, scale factors, anisotropy, shear, and deceleration parameter are given by 


$$
\begin{aligned}
\theta(t) & =\sqrt{3 \Lambda} \frac{e^{4 \sqrt{3 \Lambda}\left(t-t_{0}\right)}-\alpha^{2}+12 \Lambda \beta}{\left(e^{2 \sqrt{3 \Lambda}\left(t-t_{0}\right)}-\alpha\right)^{2}-12 \Lambda \beta}, \\
a_{i}(t) & =a_{0 i} e^{-\sqrt{\Lambda / 3}\left(t-t_{0}\right)}\left[\left(e^{2 \sqrt{3 \Lambda}\left(t-t_{0}\right)}-\alpha\right)^{2}-12 \Lambda \beta\right]^{1 / 6} \exp \left[2 K_{i} F\left(e^{\sqrt{3 \Lambda}\left(t-t_{0}\right)}\right)\right], \quad i=1,2,3, \\
A(t) & =\frac{12 K^{2} e^{2 \sqrt{3 \Lambda}\left(t-t_{0}\right)}\left[\left(e^{2 \sqrt{3 \Lambda}\left(t-t_{0}\right)}-\alpha\right)^{2}-12 \Lambda \beta\right]}{\left(e^{4 \sqrt{3 \Lambda}\left(t-t_{0}\right)}-\alpha^{2}+12 \Lambda \beta\right)^{2}}, \\
\sigma^{2}(t) & =\frac{6 K^{2} \Lambda e^{2 \sqrt{3 \Lambda}\left(t-t_{0}\right)}}{\left(e^{2 \sqrt{3 \Lambda}\left(t-t_{0}\right)}-\alpha\right)^{2}-12 \Lambda \beta}, \\
q(t) & =12 e^{2 \sqrt{3 \Lambda}\left(t-t_{0}\right)} \frac{\alpha e^{4 \sqrt{3 \Lambda}\left(t-t_{0}\right)}+\left(\alpha^{2}-12 \Lambda \beta\right)\left(\alpha-2 e^{2 \sqrt{3 \Lambda}\left(t-t_{0}\right)}\right)}{\left[e^{4 \sqrt{3 \Lambda}\left(t-t_{0}\right)}-\alpha^{2}+12 \Lambda \beta\right]^{2}}-1,
\end{aligned}
$$

where $a_{0 i}, i=1,2,3$ are arbitrary constants of integration and $F(x)=\int\left[\left(x^{2}-\alpha\right)^{2}-12 \Lambda \beta\right]^{-1 / 2} d x$.

If the cosmological constant is zero, $\Lambda=0$, the evolution of the brane universe in the extreme limit of high densities is described by

$$
\begin{aligned}
V(t)= & \frac{1}{\sqrt{\alpha}} \sqrt{\alpha^{2}\left(t-t_{0}\right)^{2}-\beta}, \\
\theta(t)= & \frac{\alpha^{2}\left(t-t_{0}\right)}{\alpha^{2}\left(t-t_{0}\right)^{2}-\beta}, \\
a_{i}(t)= & a_{0 i}\left[\alpha^{2}\left(t-t_{0}\right)^{2}-\beta\right]^{1 / 6}\left[\alpha\left(t-t_{0}\right)\right. \\
& \left.+\sqrt{\alpha^{2}\left(t-t_{0}\right)^{2}-\beta}\right]^{K_{i} / \sqrt{\alpha}}, \quad i=1,2,3, \\
A(t)= & \frac{3 K^{2}}{\alpha^{3}} \frac{\alpha^{2}\left(t-t_{0}\right)^{2}-\beta}{\left(t-t_{0}\right)^{2}}, \\
\sigma^{2}(t)= & \frac{\alpha K^{2}}{2\left[\alpha^{2}\left(t-t_{0}\right)^{2}-\beta\right]}, \\
q(t)= & 2+\frac{3 \beta}{\alpha^{2}\left(t-t_{0}\right)^{2}} .
\end{aligned}
$$

\section{SCALAR FIELD EVOLUTION ON THE ANISOTROPIC BIANCHI TYPE I BRANE}

In the previous sections we have considered the evolution of ordinary matter, obeying a barotropic equation of state, on the branelike universe. In this case the gravitational field equations can be solved exactly, with the general solution represented in an exact parametric form for arbitrary $\gamma$ and in an exact analytic form in the extreme limit of high densities, corresponding to $\gamma=2$.
As suggested by particle physics models, scalar fields, $\phi$, with energy density and pressure given by $\rho_{\phi}=\dot{\phi}^{2} / 2$ $+U(\phi)$ and $p_{\phi}=\dot{\phi}^{2} / 2-U(\phi)$, respectively, where $U(\phi)$ is the scalar field potential, are supposed to play a fundamental role in the evolution of the early universe. For a Bianchi type I brane-universe filled with a scalar field the gravitational field equations take the form

$$
\begin{aligned}
3 \dot{H}+\sum_{i=1}^{3} H_{i}^{2}= & k_{4}^{2}\left[U(\phi)-\dot{\phi}^{2}\right]-k_{5}^{4}\left[\frac{1}{6}\left(\frac{\dot{\phi}^{2}}{2}+U(\phi)\right)^{2}\right. \\
& \left.+\frac{1}{4}\left(\frac{\dot{\phi}^{4}}{4}-U^{2}(\phi)\right)\right], \\
\frac{1}{V} \frac{d}{d t}\left(V H_{i}\right)= & k_{4}^{2} U(\phi)-\frac{1}{12} k_{5}^{4}\left(\frac{\dot{\phi}^{4}}{4}-U^{2}(\phi)\right), \quad i=1,2,3 .
\end{aligned}
$$

The scalar field also obeys the following evolution equation:

$$
\ddot{\phi}+3 H \dot{\phi}+\frac{d U(\phi)}{d \phi}=0 .
$$

Depending on the functional form of the potential $U(\phi)$, several cosmological scenarios of scalar field evolution on the brane can be obtained.

As a first example we consider that the scalar field potential is a constant, $U(\phi)=\Lambda=$ const $>0$. The potential acts as a cosmological constant. Hence Eq. (46) gives $\dot{\phi}=2 \phi_{0} / V$, with $\phi_{0}>0$ a constant of integration. Adding Eqs. (45) we find the basic equation describing the dynamics of the constant potential scalar field on the brane:

$$
\ddot{V}=\lambda V-\frac{\lambda_{0}}{V^{3}},
$$

where we denoted $\lambda=3 k_{4}^{2} \Lambda+k_{5}^{4} \Lambda^{2} / 4$ and $\lambda_{0}=k_{5}^{4} \phi_{0}^{4}>0$.

The general solution of Eq. (47) is given by 
$V(t)=\frac{1}{2 \sqrt{\lambda}} e^{-\sqrt{\lambda}\left(t-t_{0}\right)} \sqrt{\left(e^{2 \sqrt{\lambda}\left(t-t_{0}\right)}-C\right)^{2}-4 \lambda_{0} \lambda}$,

with $C$ a constant of integration. This solution is similar to the solution corresponding to the stiff matter cosmological fluid and all the other physical quantities can be obtained from Eqs. (33) $-(37)$ by means of the formal substitutions $3 \Lambda \rightarrow \lambda, \alpha \rightarrow C$, and $\beta \rightarrow \lambda_{0}$. Therefore, by choosing the value of $t_{0}$ such that $\exp \left(-2 \sqrt{\lambda} t_{0}\right)=C+\sqrt{4 \lambda_{0} \lambda}$, then the brane Universe evolves from $t=0$ with a singular initial state.

As a second example of scalar field evolution on the brane we consider a scalar field with potential energy proportional to the kinetic one, so that $U(\phi)=\left(\gamma_{\phi}-1\right) \dot{\phi}^{2} / 2, \gamma_{\phi}=$ const $>1$ [29]. The energy density and pressure of the scalar field are given by $\rho_{\phi}=\gamma_{\phi} \dot{\phi}^{2} / 2$ and $p_{\phi}=\left(2-\gamma_{\phi}\right) \dot{\phi}^{2} / 2$, respectively. The evolution equation for the scalar field gives $\dot{\phi}$ $=2 \phi_{0} V^{-1 / \gamma_{\phi}}$. The evolution equation for the volume scale factor becomes

$$
\ddot{V}=6 k_{4}^{2} \phi_{0}^{2}\left(\gamma_{\phi}-1\right) V^{1-2 / \gamma_{\phi}-k_{5}^{4}} \phi_{0}^{4} \gamma_{\phi}\left(2-\gamma_{\phi}\right) V^{1-4 / \gamma_{\phi}},
$$

and has the general solution

$$
t-t_{0}=\int\left[6 k_{4}^{2} \phi_{0}^{2} \gamma_{\phi} V^{2-2 / \gamma_{\phi}}+k_{5}^{4} \phi_{0}^{4} \gamma_{\phi}^{2} V^{2-4 / \gamma_{\phi}+C}\right]^{-1 / 2} d V,
$$

with $t_{0}$ and $C$ arbitrary constants of integration. The time variation of the physically important parameters is given in a parametric form, with $V$ taken as parameter, by

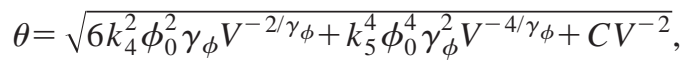

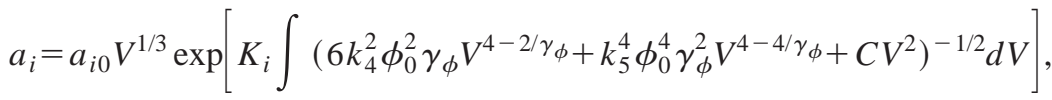

$$
\begin{aligned}
& A=\frac{3 K^{2}}{6 k_{4}^{2} \phi_{0}^{2} \gamma_{\phi} V^{2-2 / \gamma_{\phi}}+k_{5}^{4} \phi_{0}^{4} \gamma_{\phi}^{2} V^{2-4 / \gamma_{\phi}+C}}, \\
& \sigma^{2}=\frac{K^{2}}{2} V^{-2}
\end{aligned}
$$

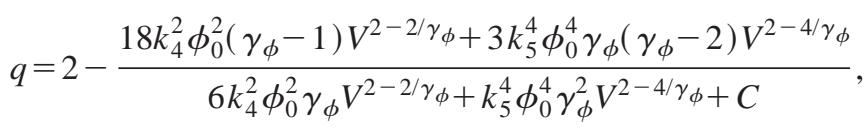

$$
\begin{aligned}
& \phi-\phi_{0}^{\prime}=2 \phi_{0} \int\left[6 k_{4}^{2} \phi_{0}^{2} \gamma_{\phi} V^{2-2 / \gamma_{\phi}}+k_{5}^{4} \phi_{0}^{4} \gamma_{\phi}^{2} V^{2-4 / \gamma_{\phi}}+C\right]^{-1 / 2} V^{-1 / \gamma_{\phi}} d V,
\end{aligned}
$$

with $a_{i 0}, i=1,2,3$ and $\phi_{0}^{\prime}$ constants of integration. In this model the scalar field satisfies an equation of state of the form $p_{\phi}=\left(2 / \gamma_{\phi}-1\right) \rho_{\phi}$.

The cosmological behavior of universes filled with a scalar field, $\phi$, as well as a Liouville type exponential potential $U(\phi)=\Lambda e^{-k \phi}, \Lambda, k=$ const $>0$, has been extensively investigated in the physical literature for both homogeneous and inhomogeneous scalar fields (for a discussion of the physical relevance of the exponential potential see Ref. [30], and references therein). The exponential type potential arises in the four-dimensional effective Kaluza-Klein type theories from compactification of the higher-dimensional supergravity or superstring theories. In string or Kaluza-Klein theories the moduli fields associated with the geometry of the extra dimensions may have effective exponential potentials due to the curvature of the internal spaces or to the interaction of the moduli with form fields on the internal spaces. Exponential potentials can also arise due to nonperturbative effects such as gaugino condensation.

For the flat isotropic Friedmann-Robertson-Walker geometry a particular solution of the field equations with $\phi$ $=\delta \ln t$, has been obtained by Barrow [31]. In the framework of general relativity there is no anisotropic Bianchi type I solution of this type.

We try to generalize this solution for the case of the scalar fields confined on the brane. By assuming that the constants $k$ and $\delta$ satisfy the condition $k \delta=2$, it is easy to check by direct calculations that the following expressions satisfy the field equations (44)-(46):

$$
V(t)=V_{0} t^{2}, \quad H(t)=\frac{2}{3} t^{-1},
$$




$$
\begin{aligned}
& a_{i}(t)=a_{i 0} t^{2 / 3} \exp \left(-K_{i} V_{0}^{-1} t^{-1}\right), \quad i=1,2,3 \\
& A(t)=\frac{3}{4} K^{2} V_{0}^{-2} t^{-2}, \quad \sigma^{2}(t)=\frac{1}{2} K^{2} V_{0}^{-2} t^{-4}, \quad q(t)=\frac{1}{2} \\
& \phi(t)=\frac{2}{\sqrt{3} k_{4}} \ln t, \quad U(t)=\frac{2}{3 k_{4}^{2}} t^{-2}
\end{aligned}
$$

The scalar field and the potential are determined by the four-dimensional coupling constant, with $\Lambda=2 / 3 k_{4}^{2}, k$ $=\sqrt{3} k_{4}$ and $\delta=2 / \sqrt{3} k_{4}$. The integrations constants $K_{i}, i$ $=1,2,3$ satisfy the consistency conditions $\sum_{i=1}^{3} K_{i}=0$.

However, if we substitute above results to Eq. (44), then we will find a constraint on the constants of integration $K^{2}$ $=-8 k_{5}^{4} V_{0}^{2} / 27 k_{4}^{4}$. From the expression of $K^{2}$ we can see that for the general relativistic case, $k_{5}=0$, only isotropic solutions can exist, namely $K^{2}=\sum_{i=1}^{3} K_{i}^{2}=0$, leading to $K_{i}$ $\equiv 0, \forall i$ and to a zero pressure scalar field, $p_{\phi} \equiv 0$. For the brane world model, the constraint condition for $K^{2}$ indicates that the scalar field driven brane universe with exponential type potential and with a simple logarithmic time dependence of the scalar field cannot exist, in general.
In the case of this simple model the scalar field potential can also be given, in the isotropic case, as a function of the volume scale factor, $U \sim V^{-1}$. It is interesting to find the general solution of the field equations for this choice of $U$ for the anisotropic brane, without imposing any supplementary restrictions on the physical variables. For the sake of generality we consider a more general problem, with a general functional dependence of the scalar field potential on the volume scale factor $U=U(V)$. With this assumption the Klein-Gordon equation for the scalar field (46) can be integrated to give

$$
\dot{\phi}^{2}=\frac{2}{V^{2}}\left(B-\int V^{2} \frac{d U}{d V} d V\right)
$$

and

$$
\rho_{\phi}=\frac{\dot{\phi}^{2}}{2}+U=\frac{1}{V^{2}}[B+Y(V)],
$$

where $B$ is a constant of integration and $Y(V)$ $=2 \int V U(V) d V$. The general solution of the gravitational field equations on the brane can be expressed again in an exact parametric form, with $V$ as parameter:

$$
\begin{aligned}
& t-t_{0}=\int\left\{\frac{3}{2} K^{2}+3 k_{4}^{2}[B+Y(V)]+\frac{k_{5}^{4}}{4} V^{-2}[B+Y(V)]^{2}\right\}^{-1 / 2} d V, \\
& \theta=\sqrt{\frac{3}{2} K^{2} V^{-2}+3 k_{4}^{2} V^{-2}[B+Y(V)]+\frac{k_{5}^{4}}{4} V^{-4}[B+Y(V)]^{2}}, \\
& a_{i}=a_{i 0} V^{1 / 3} \exp \left\{K_{i} \int\left[\frac{3}{2} K^{2} V^{2}+3 k_{4}^{2} V^{2}[B+Y(V)]+\frac{k_{5}^{4}}{4}[B+Y(V)]^{2}\right]^{-1 / 2} d V\right\}, \quad i=1,2,3, \\
& A=\frac{3 K^{2}}{\frac{3}{2} K^{2}+3 k_{4}^{2}[B+Y(V)]+\frac{k_{5}^{4}}{4} V^{-2}[B+Y(V)]^{2}}, \\
& \sigma^{2}=\frac{K^{2}}{2 V^{2}} \\
& q=2-\frac{18 k_{4}^{2} V Y^{\prime}(V)+3 k_{5}^{4} V^{-1}[B+Y(V)] Y^{\prime}(V)-3 k_{5}^{4} V^{-2}[B+Y(V)]^{2}}{6 K^{2}+12 k_{4}^{2}[B+Y(V)]+k_{5}^{4} V^{-2}[B+Y(V)]^{2}}, \\
& \phi-\phi_{0}=\int \frac{\sqrt{2 B+2 Y(V)-V Y^{\prime}(V)} d V}{\sqrt{\frac{3}{2} K^{2} V^{2}+3 k_{4}^{2}[B+Y(V)] V^{2}+\frac{k_{5}^{4}}{4}[B+Y(V)]^{2}}},
\end{aligned}
$$


with $t_{0}, a_{i 0}, i=1,2,3$ and $\phi_{0}$ constants of integration and $Y^{\prime}(V)=d Y(V) / d V$.

Suppose that the scalar field potential is given, as a function of the volume scale factor, by the simple relation $U$ $=u_{0} / V$, with $u_{0}>0$ a constant. Then, by denoting $\omega$ $=3 k_{4}^{2} u_{0} / 2$ and $\tau=\left(3 K^{2}+2 k_{5}^{4} u_{0}^{2}\right) / 12 k_{4}^{2} u_{0}$ and taking the integration constant $B=0$, the general solution of the gravitational field equations for the scalar field confined on the anisotropic brane take the form

$V(t)=\omega\left(t-t_{0}\right)^{2}-\tau, \quad \theta(t)=\frac{2 \omega\left(t-t_{0}\right)}{\omega\left(t-t_{0}\right)^{2}-\tau}$,

$a_{i}(t)=a_{i 0} \prod_{j=1,2}\left[\sqrt{\omega}\left(t-t_{0}\right)+\varepsilon_{j} \sqrt{\tau}\right]^{1 / 3-\varepsilon_{j} K_{i} / 2 \sqrt{\omega \tau}}, \quad i=1,2,3$,

$A(t)=\frac{3 K^{2}}{4 \omega^{2}\left(t-t_{0}\right)^{2}}, \sigma^{2}(t)=\frac{K^{2}}{2\left[\omega\left(t-t_{0}\right)^{2}-\tau\right]^{2}}$,

$q(t)=\frac{1}{2}+\frac{3 \tau}{2 \omega\left(t-t_{0}\right)^{2}}$,

$\phi(t)=\frac{2}{\sqrt{3} k_{4}} \sinh ^{-1}\left[\sqrt{\frac{\omega}{\tau}\left(t-t_{0}\right)^{2}-1}\right]$,

$U(t)=\frac{u_{0}}{\omega\left(t-t_{0}\right)^{2}-\tau}, \quad U(\phi)=\frac{u_{0}}{\tau} \sinh ^{-2}\left(\frac{\sqrt{3} k_{4}}{2} \phi\right)$,

where we have also taken $\phi_{0}=0$ and denoted $\varepsilon_{1}=+1, \varepsilon_{2}$ $=-1$.

\section{PERFECT FLUID BIANCHI TYPE V BRANE COSMOLOGIES}

The line element of a Bianchi type V space-time, representing the anisotropic generalization of the open $k=-1$ Robertson-Walker geometry, is given by

$$
d s^{2}=-d t^{2}+a_{1}^{2}(t) d x^{2}+a_{2}^{2}(t) e^{-2 x} d y^{2}+a_{3}^{2}(t) e^{-2 x} d z^{2} .
$$

The effective Einstein field equations on the brane are given by

$$
3 \dot{H}+\sum_{i=1}^{3} H_{i}^{2}=\Lambda-\frac{3 \gamma-2}{2} k_{4}^{2} \rho-\frac{3 \gamma-1}{12} k_{5}^{4} \rho^{2},
$$

$$
\begin{aligned}
\frac{1}{V} \frac{d}{d t}\left(V H_{i}\right)-2 a_{1}^{-2} & =\Lambda-\frac{\gamma-2}{2} k_{4}^{2} \rho-\frac{\gamma-1}{12} k_{5}^{4} \rho^{2}, \quad i=1,2,3 \\
2 H_{1}-H_{2}-H_{3} & =0, \\
\dot{\rho}+3 \gamma H \rho & =0 .
\end{aligned}
$$

From Eq. (77) we obtain $a_{2} a_{3}=a_{1}^{2}$, leading to $V=a_{1}^{3}$. The distribution of the matter energy density on the brane is given again by $\rho=\rho_{0} V^{-\gamma}$. Adding Eqs. (76) we obtain

$$
\frac{1}{V} \frac{d}{d t}(V H)=\Lambda+2 V^{-2 / 3}-\frac{\gamma-2}{2} k_{4}^{2} \rho-\frac{\gamma-1}{12} k_{5}^{4} \rho^{2} .
$$

Subtracting Eq. (79) from Eqs. (76) leads again to

$$
H_{i}=H+\frac{K_{i}}{V}, \quad i=1,2,3
$$

with $K_{i}, i=1,2,3$ constants of integration satisfying the consistency condition $\sum_{i=1}^{3} K_{i}=0$.

Substitution of the Hubble parameters $H_{i}, \quad i=1,2,3$ into Eq. (77) gives $2 K_{1}=K_{2}+K_{3}$ and, consequently, we obtain

$$
K_{1}=0, \quad K_{2}=-K_{3}
$$

The general dynamics of the Bianchi type $\mathrm{V}$ space-time is described by the equation

$$
\ddot{V}=6 V^{1 / 3}+3 \Lambda V-\frac{3(\gamma-2)}{2} k_{4}^{2} \rho_{0} V^{1-\gamma}-\frac{\gamma-1}{4} k_{5}^{4} \rho_{0}^{2} V^{1-2 \gamma},
$$

with the general solution

$$
\begin{aligned}
t-t_{0}= & \int\left(9 V^{4 / 3}+3 \Lambda V^{2}+3 k_{4}^{2} \rho_{0} V^{2-\gamma}\right. \\
& \left.+\frac{1}{4} k_{5}^{4} \rho_{0}^{2} V^{2-2 \gamma}+C\right)^{-1 / 2} d V,
\end{aligned}
$$

where $C$ is a constant of integration.

Therefore for a Bianchi type $\mathrm{V}$ induced brane geometry the general solution of the gravitational field equations can be expressed in the following exact parametric form, with $V \geqslant 0$ taken as parameter: 


$$
\begin{aligned}
\theta & =\left(9 V^{-2 / 3}+3 \Lambda+3 k_{4}^{2} \rho_{0} V^{-\gamma}+\frac{1}{4} k_{5}^{4} \rho_{0}^{2} V^{-2 \gamma}+C V^{-2}\right)^{1 / 2}, \\
a_{i} & =a_{0 i} V^{1 / 3} \exp \left[K_{i} \int\left(9 V^{10 / 3}+3 \Lambda V^{4}+3 k_{4}^{2} \rho_{0} V^{4-\gamma}+\frac{1}{4} k_{5}^{4} \rho_{0}^{2} V^{4-2 \gamma}+C V^{2}\right)^{-1 / 2} d V\right], \quad i=1,2,3, \\
A & =3 K^{2}\left(9 V^{4 / 3}+3 \Lambda V^{2}+3 k_{4}^{2} \rho_{0} V^{2-\gamma}+\frac{1}{4} k_{5}^{4} \rho_{0}^{2} V^{2-2 \gamma}+C\right)^{-1}, \\
\sigma^{2} & =\frac{K^{2}}{2} V^{-2}, \\
q & =2-\frac{72 V^{4 / 3}+36 \Lambda V^{2}+18(2-\gamma) k_{4}^{2} \rho_{0} V^{2-\gamma}+3(1-\gamma) k_{5}^{4} \rho_{0}^{2} V^{2-2 \gamma}}{36 V^{4 / 3}+12 \Lambda V^{2}+12 k_{4}^{2} \rho_{0} V^{2-\gamma}+k_{5}^{4} \rho_{0}^{2} V^{2-2 \gamma}+4 C}
\end{aligned}
$$

where $K^{2}=\sum_{i=1}^{3} K_{i}^{2}$.

Substitution of the Hubble parameter and of the density into Eq. (75) gives the second consistency condition satisfied by the constants $K_{i}, \quad i=2,3$ :

$$
K^{2}=\frac{2}{3} C \text {. }
$$

Hence we can express the integration constants $K_{2}, K_{3}$ as functions of the constant $C$ :

$$
K_{2}= \pm \sqrt{\frac{C}{3}}, \quad K_{3}=\mp \sqrt{\frac{C}{3}}
$$

In the limit of high matter densities $(\gamma=2)$ the general solution of the gravitational field equations for a Bianchi type $\mathrm{V}$ geometry cannot be expressed in an exact analytic form.

\section{DISCUSSIONS AND FINAL REMARKS}

In the present paper we have considered some classes of exact solutions of the gravitational field equations on the brane world in the framework of the Randall-Sundrum scenario. The Randall-Sundrum mechanism was originally motivated as a possible mechanism for evading Kaluza-Klein compactification by localizing gravity in the presence of an uncompactified extra dimension. In this model, the 5-dimensional bulk space-time is assumed to be vacuum except for the presence of a cosmological constant. Matter fields on the brane are regarded as responsible for the dynamics of the brane. The quadratic terms in the energymomentum tensor, due to the form of the Gauss-Codazzi equations, may be important in the very early stages of the Universe, leading to major changes in the dynamics of the Universe.

For a perfect linear barotropic cosmological fluid confined on the brane, the early cosmological evolution in anisotropic Bianchi type I and $\mathrm{V}$ geometries is fundamentally changed by the inclusion of the terms proportional to the square of the energy density. The time variation of the mean anisotropy parameter of the Bianchi type I space-time is represented, for different values of $\gamma$, in Fig. 1. At high densities the brane Universe starts its evolution from an isotropic state, with $A\left(t_{0}\right)=0$. The anisotropy increases and reaches a maximum value after a finite time interval $t_{\max }$, and for $t>t_{\max }$, the mean anisotropy is a monotonically decreasing function, tending to zero in the large time limit. This behavior is in sharp contrast to the usual general relativistic evolution, illustrated by the case $\gamma=1$ in Fig. 1 (for this choice the quadratic contribution to the energy-momentum tensor vanishes), in which the Universe is born in a state of maximum anisotropy. In the initial stage the evolution is noninflationary, but in the large time limit the brane universe ends in an inflationary stage. In Fig. 2 we present the dynamics of the deceleration parameter for different values of $\gamma$. In the limit of small $t$ (and small $V$, also), and with $C=0$, from Eq. (23) we obtain $V \sim t^{1 / \gamma}$ (this result is valid for both Bianchi type I and $\mathrm{V}$ geometries). Therefore in the early stages of evolution of the Universe the mean anisotropy varies as $A \sim t^{2-2 / \gamma}$. Near the singular point the scale factors behave as $a_{i}$ $\sim t^{1 / 3 \gamma} \exp \left[\gamma /(1+\gamma) K_{i}^{\prime} t^{1+1 / \gamma}\right]$. The deceleration parameter is

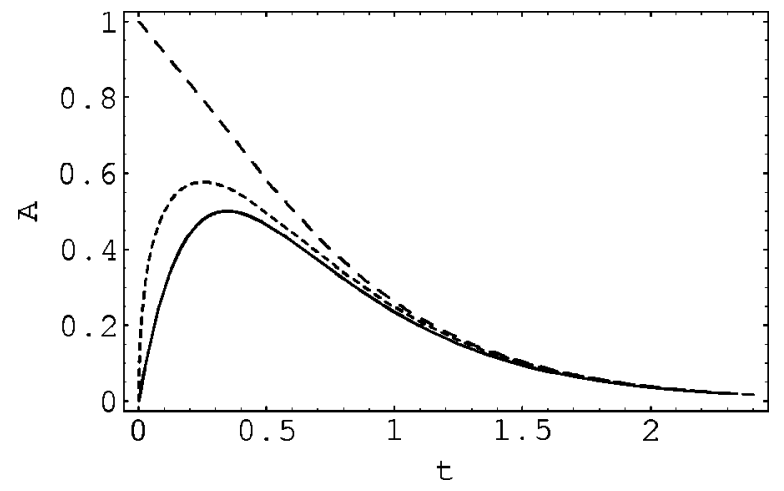

FIG. 1. Variation as a function of time of the anisotropy parameter of the Bianchi type I brane universe with confined perfect cosmological fluid for $\gamma=2$ (solid curve), $\gamma=4 / 3$ (dotted curve), and $\gamma=1$ (dashed curve). The constants have been normalized according to the conditions $3 \Lambda=1,3 k_{4}^{2} \rho_{0}=1, k_{5}^{4} \rho_{0}^{2} / 4=1, C=1$, and $K^{2}=2 / 3$. 
given by $q=3 \gamma-1$. Figures 3 and 4 present the time evolution of the mean anisotropy and deceleration parameter for the brane with Bianchi type V geometry, the general features of the cosmological dynamics being similar to the Bianchi type I case.

The general evolution of scalars fields confined on the anisotropic brane is also modified by the presence of the quadratic terms in the energy-momentum tensor. For a constant potential scalar field, the solution of the gravitational field equations is very similar to the stiff cosmological fluid case, with the cosmic evolution starting from an isotropic state, with zero anisotropy and with a maximum anisotropic phase reached after a finite time $t_{\max }$. The dynamics of the scalar field satisfying the equation of state $p_{\phi}=\left(2 / \gamma_{\phi}\right.$ -1) $\rho_{\phi}, \quad \gamma_{\phi}>1$, depends on the values of $\gamma_{\phi}$. For $1<\gamma_{\phi}$ $<3 / 2$, the Universe is born in an isotropic state, but for $\gamma_{\phi}$ $>3 / 2$ the behavior is similar to the general relativistic case, with an infinite initial anisotropy, which rapidly decays to zero. For small $\gamma_{\phi}$ the evolution is noninflationary. In the limit of large $\gamma_{\phi}$ the scalar field behaves as a cosmological fluid obeying an equation of state of the form $p_{\phi}=-\rho_{\phi}$. In this case and for large times the behavior of the Universe is described by

$$
\begin{aligned}
& V=V_{0} \exp \left(H_{0} t\right), \quad H=\frac{1}{3} H_{0}, \\
& a_{i}=a_{i 0} \exp \left(\frac{H_{0}}{3} t-\frac{K_{i}}{V_{0} H_{0}} e^{-H_{0} t}\right), \quad i=1,2,3, \\
& A=\frac{3 K^{2}}{V_{0}^{2} H_{0}^{2}} \exp \left(-2 H_{0} t\right), \quad q=-1, \quad \phi=2 \phi_{0} t, \\
& \rho_{\phi}=2 \phi_{0}^{2} \gamma_{\phi}=\text { const },
\end{aligned}
$$

where $H_{0}=\phi_{0} \sqrt{6 k_{4}^{2} \gamma_{\phi}+k_{5}^{4} \phi_{0}^{2} \gamma_{\phi}^{2}}$. In the large time limit the Universe ends in an isotropic de Sitter inflationary phase.

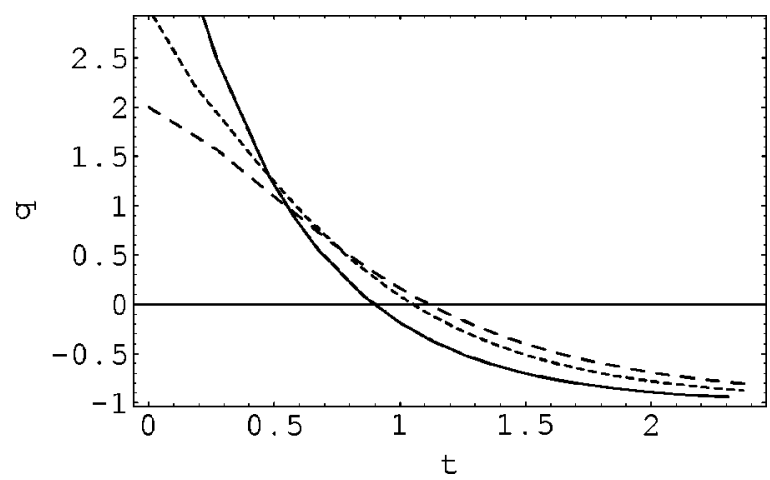

FIG. 2. Variation as a function of the time of the deceleration parameter $q$ of the Bianchi type I brane geometry, with perfect cosmological fluid confined on the brane, for $\gamma=2$ (solid curve), $\gamma=4 / 3$ (dotted curve), and for $\gamma=1$ (dashed curve). The constants have been normalized according to the conditions $3 \Lambda=1,3 k_{4}^{2} \rho_{0}$ $=1, k_{5}^{4} \rho_{0}^{2} / 4=1$, and $C=1$.

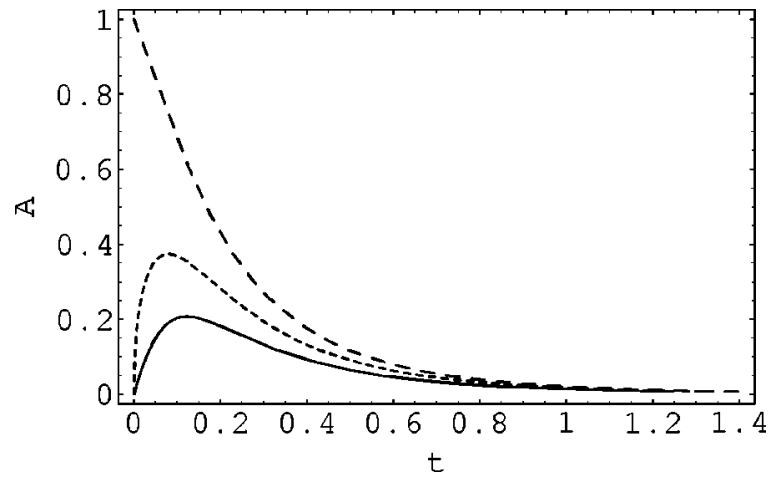

FIG. 3. Variation as a function of time of the anisotropy parameter of the Bianchi type $\mathrm{V}$ brane universe with confined perfect cosmological fluid for $\gamma=2$ (solid curve), $\gamma=4 / 3$ (dotted curve), and $\gamma=1$ (dashed curve). The constants have been normalized according to the conditions $3 \Lambda=1,3 k_{4}^{2} \rho_{0}=1, k_{5}^{4} \rho_{0}^{2} / 4=1, C=1$, and $K^{2}=2 / 3$.

The time variations of the anisotropy and deceleration parameters are represented, for different values of $\gamma_{\phi}$, in Figs. 5 and 6.

Scalar fields with potentials of the form $U(\phi)$ $=U_{0} \sinh ^{\beta}(\alpha \phi), U_{0}, \beta, \alpha=$ const, have been recently considered in quintessence models as tracker solutions of the gravitational field equations, which can drive the Universe in its present nondecelerating phase [32]. For such a type of potential we have obtained the general solution of the field equations in the case of the anisotropic brane world for $\beta$ $=-2$. The asymptotic behavior of the potential corresponds to an inverse power-law-like form for small $\phi, U(\phi)$ $\sim \phi^{-2}$ and to an exponential one for large values of the scalar field $U(\phi) \sim e^{-\sqrt{3} k_{4} \phi}$. In this model the Universe starts from a state with infinite anisotropy and ends in an isotropic phase, but the evolution is generally noninflationary, with $q$ $>0$ for all times.

It is interesting to note that in two measure gravitational theories with scale invariance a dilaton field with exponential type potentials has to be also introduced, leading to non-

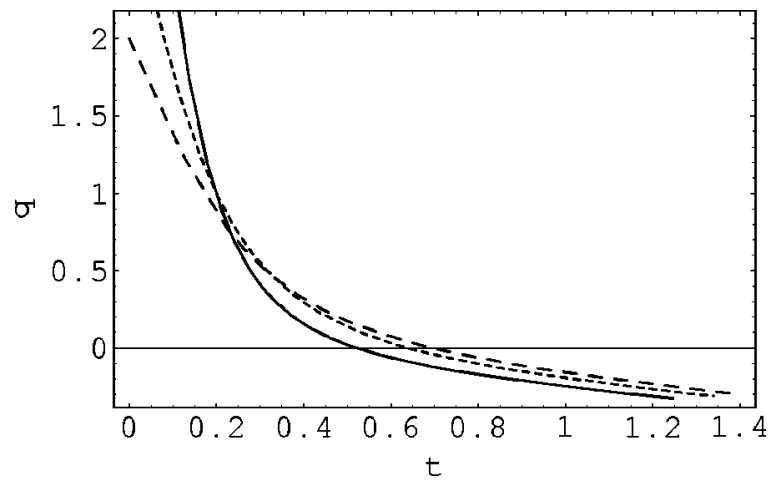

FIG. 4. Variation as a function of the time of the deceleration parameter $q$ of the Bianchi type $\mathrm{V}$ brane geometry, with perfect cosmological fluid confined on the brane, for $\gamma=2$ (solid curve), $\gamma=4 / 3$ (dotted curve), and for $\gamma=1$ (dashed curve). The constants have been normalized according to the conditions $3 \Lambda=1,3 k_{4}^{2} \rho_{0}$ $=1, k_{5}^{4} \rho_{0}^{2} / 4=1$, and $C=1$. 


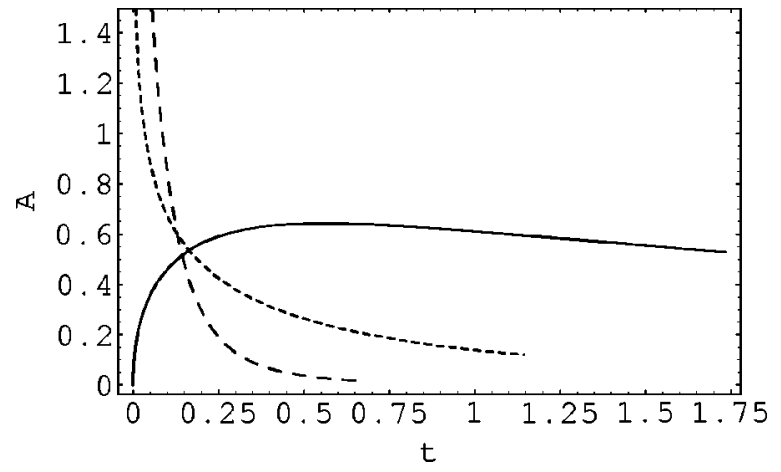

FIG. 5. Variation as a function of time of the anisotropy parameter of the Bianchi type I brane universe with confined scalar fields obeying the equation of state $p_{\phi}=\left(2 / \gamma_{\phi}-1\right) \rho_{\phi}$ for $\gamma_{\phi}=1.5$ (solid curve), $\gamma_{\phi}=2$ (dotted curve), and $\gamma_{\phi}=5$ (dashed curve). The constants have been normalized according to the conditions $6 k_{4}^{2} \phi_{0}^{2}$ $=1, k_{5}^{4} \phi_{0}^{4}=1, C=1$, and $K^{2}=1$.

trivial mass generation. Interpolating models for natural transition from inflation to a slowly accelerated universe at late times appear naturally [22]. For cosmological models without the cosmological constant problem and theories of extended objects (strings, branes) without a fundamental scale see Guendelman [33,34].

We have not presented the solutions corresponding to scalar fields confined to the Bianchi type V brane world. Their mathematical form is very similar to the Bianchi type I case, with one correction term, proportional to $V^{4 / 3}$ added to the parametric time equation. Due to the presence of this term,

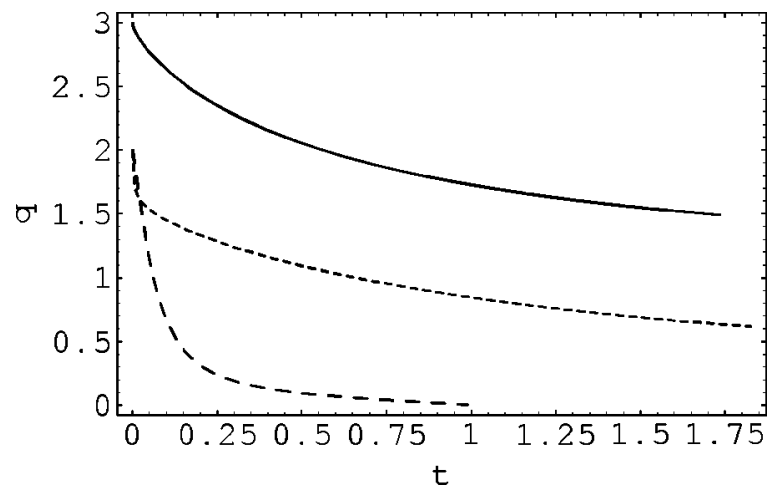

FIG. 6. Variation as a function of time of the deceleration parameter $q$ of the Bianchi type I brane universe with confined scalar fields obeying the equation of state $p_{\phi}=\left(2 / \gamma_{\phi}-1\right) \rho_{\phi}$ for $\gamma_{\phi}=1.5$ (solid curve), $\gamma_{\phi}=2$ (dotted curve), and $\gamma_{\phi}=5$ (dashed curve). The constants have been normalized according to the conditions $6 k_{4}^{2} \phi_{0}^{2}=1, k_{5}^{4} \phi_{0}^{4}=1$, and $C=1$.

exact analytic solutions cannot be generally obtained for scalar field models in Bianchi type V space-times, but the general physical behavior of scalar fields in both geometries has the same qualitative features.

\section{ACKNOWLEDGMENTS}

The work of C.M.C. is supported by the Taiwan CosPA project and, in part, by the Center of Theoretical Physics at NTU and National Center for Theoretical Science.
[1] L. Randall and R. Sundrum, Phys. Rev. Lett. 83, 3370 (1999).

[2] L. Randall and R. Sundrum, Phys. Rev. Lett. 83, 4690 (1999).

[3] P. Horava and E. Witten, Nucl. Phys. B460, 506 (1996).

[4] H. B. Kim and H. D. Kim, Phys. Rev. D 61, 064003 (2000).

[5] P. Binetruy, C. Deffayet, U. Ellwanger, and D. Langlois, Phys. Lett. B 477, 285 (2000).

[6] P. Binetruy, C. Deffayet, and D. Langlois, Nucl. Phys. B565, 269 (2000).

[7] H. Stoica, S.-H. H. Tye, and I. Wasserman, Phys. Lett. B 482, 205 (2000).

[8] D. Langlois, R. Maartens, and D. Wands, Phys. Lett. B 489, 259 (2000).

[9] H. Kodama, A. Ishibashi, and O. Seto, Phys. Rev. D 62, 064022 (2000).

[10] C. van de Bruck, M. Dorca, R. H. Brandenberger, and A. Lukas, Phys. Rev. D 62, 123515 (2000).

[11] C. Csaki, J. Erlich, T. J. Hollowood, and Y. Shirman, Nucl. Phys. B581, 309 (2000).

[12] R. Maartens, "Geometry and dynamics of the brane-world," gr-qc/0101059.

[13] C. Csaki, M. Graesser, C. Kolda, and J. Terning, Phys. Lett. B 462, 34 (1999).

[14] J. M. Cline, C. Grojean, and G. Servant, Phys. Rev. Lett. 83, 4245 (1999).
[15] L. Anchordoqui, C. Nuñez, and K. Olsen, J. High Energy Phys. 10, 050 (2000).

[16] T. Shiromizu, K. Maeda, and M. Sasaki, Phys. Rev. D 62, 024012 (2000).

[17] M. Sasaki, T. Shiromizu, and K. Maeda, Phys. Rev. D 62, 024008 (2000).

[18] K. Maeda and D. Wands, Phys. Rev. D 62, 124009 (2000).

[19] E. I. Guendelman, Mod. Phys. Lett. A 14, 1043 (1999).

[20] E. I. Guendelman and A. B. Kaganovich, Phys. Rev. D 60, 065004 (1999).

[21] E. I. Guendelman, Class. Quantum Grav. 17, 361 (2000).

[22] E. I. Guendelman, "The volume element of space-time and scale invariance," hep-th/0011049.

[23] R. Maartens, Phys. Rev. D 62, 084023 (2000).

[24] R. Maartens, V. Sahni, and T. D. Saini, Phys. Rev. D 63, 063509 (2001).

[25] A. V. Toporensky, "The shear dynamics in Bianchi type I cosmological model on the brane," gr-qc/0103093.

[26] A. Campos and C. F. Sopuerta, Phys. Rev. D 63, 104012 (2001).

[27] A. V. Frolov, "Kasner-AdS spacetime and anisotropic braneworld cosmology," gr-qc/0102064.

[28] M. G. Santos, F. Vernizzi, and P. G. Ferreira, "Isotropisation and instability of the brane," hep-ph/0103112.

[29] J. Barrow and P. Saich, Class. Quantum Grav. 10, 279 (1993). 
[30] A. A. Coley, "Dynamical systems in cosmology," gr-qc/9910074.

[31] J. Barrow, Phys. Lett. B 187, 12 (1987).

[32] L. A. Urena-Lopez and T. Matos, Phys. Rev. D 62, 081302(R) (2000).
[33] E. I. Guendelman, "Measure fields, the cosmological constant and scale invariance," hep-th/0008122.

[34] E. I. Guendelman, "Scale invariance, inflation and the present vacuum energy of the Universe," gr-qc/0004011. 